\title{
Automated Aeroponics System Using IoT for Smart Farming
}

\author{
Stephen C. Kerns \\ Joong-Lyul Lee \\ Department of Mathematics and Computer Science \\ University of North Carolina at Pembroke, Pembroke, NC
}

Doi: 10.19044/esj.2017.c1p10 URL:http://dx.doi.org/10.19044/esj.2017.c1p10

\begin{abstract}
In recent years, the Internet of Things (IoT) has received much attention in the areas of industry and academia. Currently, IoT technologies are being applied in many fields and is changing lives in many areas such as smart homes, smart cities, smart grids, autonomous cars, and the industrial internet. Howbeit, traditional agriculture is still waiting for many changes to occur in networking technology especially in IoT. Many researchers and engineers are working towards applying IoT technology to traditional agricultural methods. Aeroponics farming is an efficient and effective process for growing plants without using soil. When we apply IoT technology to an aeroponics system, it is expected that there will be many improvements such as decreasing water usage, increasing plant yield, minimizing rate of growth and reducing the workforce. In this paper, we designed and implemented a new automatic aeroponics system using IoT devices. Our system is comprised of three main components: a mobile application, service platform and IoT devices with sensors. The mobile application provides the user a graphical user interface to monitor and adjust the aeroponics system. The service platform is a middleware system that provides information for the mobile application to store the gathered information from IoT devices using sensors within the aeroponics system. The IoT device uses sensors within the aeroponics system to control each pump and access data. Our work is a new application in the agricultural industry and is expected to be a promising application that will help the farmer with increasing productivity in farming and reducing carbon footprint.
\end{abstract}

Keywords: IoT (Internet of Things), Wireless Sensor Networks, Sensor Data Integration, Smart Farming, Automated Aeroponic System 


\section{Introduction}

As technology develops, it will bring great advantages to human beings in all areas of life. Especially, the development of network technology is changing many areas of our lives such as smart homes, smart cities, smart grids, autonomous cars, and the industrial internet. Smart agriculture systems are an area that would benefit from this technology. By adapting the recent innovations in technology for farming it would add many benefits for farmers and reduce our carbon footprint.

The Internet of Things (IoT) [1] is an emerging technology that provides internet connectivity and powerful data analytic capabilities to create a system in which objects in the physical world could be connected to the internet by sensors. However, smart farming is still in its infancy in regard to using IoT devices for agriculture. Aeroponics farming is an efficient and effective process for growing plants without using soil as a medium as well as with little water. In this paper, we propose new automated aeroponics system using IoT devices that will help the farmer by increasing productivity in farming. To the best of our knowledge, this is first work to design and implement of automated aeroponics system using IoT devices.

The rest of the paper is organized as follows. Section II describes current research work in smart farming systems with IoT devices and IoT applications. In section III, we present our design and implementation of automatic aeroponics systems using IoT devices for smart farming. Finally, we summarize our research work in section IV.

\section{Related Work}

In relation to IoT technology, many researchers have proposed many different applications for a smart farming system [2] [10]. We will describe some of the proposed research work in the following.

A connected farm based on IoT systems for a smart farming system was recently proposed by a researcher in Korea Electronic Technology Institute [2]. The proposed connected farm system has three main components such as connected IoT devices, an IoT gateway, and an IoT service platform. Many physical sensors and controls will provide the means for monitoring and controlling of the environmental conditions of the farm. These sensors and controllers are connected to an IoT gateway. By smartphone application, the end user can monitor and control in the farming system remotely.

An intelligent monitoring platform framework and system structure for a facility agricultural ecosystem based on IoT described in the research work [3]. The described framework is divided into four function layers: the sensor layer, transmission layer, control layer and application layer. The sensor layer handles the numerical sensor values in farming. The transmission layer processes collected data from sensor layer. The control layer uses the collected 
data to control the agricultural production by an automatic control algorithm. The application layer displays the specific business logic of agriculture by using an interactive interface. The proposed framework applies to an intelligent monitoring platform of facility habits in Shanghai.

Agri-IoT, an IoT-based framework based on semantic web technologies presented in the research work [4]. The presented system enables large-scale data processing, analysis and automatic reasoning based on realtime streams of data coming from various heterogeneous sensors, social media, connected farms, governmental alerts and regulations. The authors evaluated the proposed Agri-IoT with two realistic scenarios and investigated the introduction of smart farming using IoT and its opportunities.

A smart greenhouse using Intel Galileo Gen 2 and Arduino will help the farmers carry out the work in a farm automatically proposed by the researcher [5]. They proposed the smart greenhouse can reduce 70\% 80\% of the water requirement, increase yield and rate of growth, and produce organic agricultural products.

The design of a smart IoT communication system manager used as a low-cost irrigation controller described in the research work [6]. The authors used LoRa WAN network protocol to show the best solution for data acquisition in farming systems. The proposal is a great advantage for controlling irrigation events to reduce the amount of energy and water used.

In the survey [7], the author reviewed many IoT solutions from context-aware computing perspective into five different categories: smart wearable, smart home, smart city, smart environment, and smart enterprise.

A smart farming system using unmanned aerial vehicles is proposed by P. Lottes et al. [8]. The authors described the proposed system for vegetation detection, plant-tailored feature extraction, and classification to obtain an estimate of the distribution of crops and weed relying on objectfeatures and key points in combination with random forest and showed the classification system achieve good performance.

P. Nintanavongsa et al. [9] designed two routing approaches, called Location-agnostic and Location-specific protocols, to facilitate the selfsustaining agricultural monitoring platform, requiring no infrastructure installation, comprises of Unmanned Aerial Vehicle (UAV) with solar energy harvesting and wireless power transfer capability.

Low-cost wireless system to reduce the pesticide dosage for smart farming proposed researcher [10]. As the pesticide control is important issue in farming, the author proposed the pesticide dosage reduction using the wireless sensor network technology and the fuzzy logic theory. The proposed system has been evaluated in real test field experimentally and shown a significant reduction (47\%) of the total pesticide dosage in experiment result. 
In this research work, we introduce a new application in an automated aeroponics system using IoT for smart farming system.

\section{Design and Implementation of Aeroponics System}

In this section, we introduce our aeroponics system using IoT devices including its three main components, mobile interface, service platform, and IoT devices with each sensor.
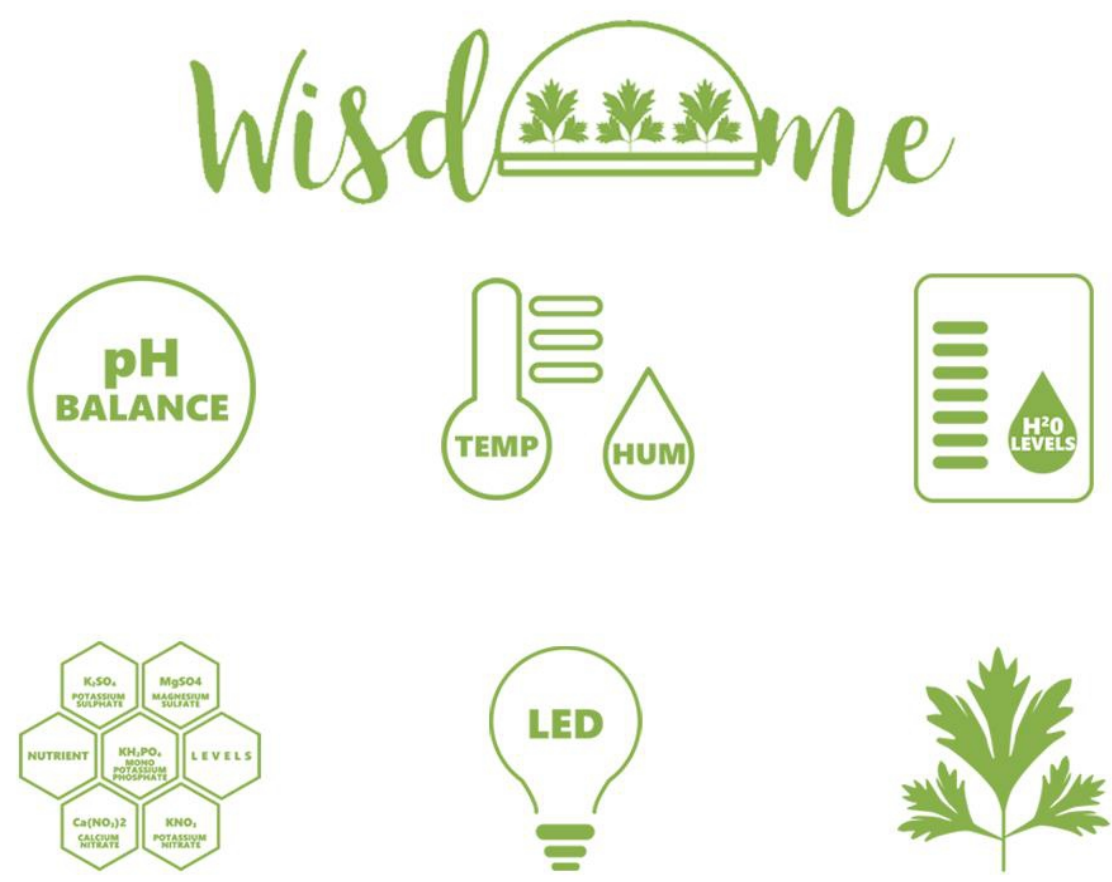

Figure 1. Graphic user interface for mobile application

\section{Mobile application}

We designed a mobile interface that a user can easily check every sensor's value in each aeroponics system through a service platform. This service platform stores the sensor's data such as temperature, humidity, $\mathrm{pH}$ balance, nutrient levels, LED lights and water level. Referencing figure 1, the user can view the current sensors' data on the service platform of the aeroponics system. For the service platform, the current web technologies HTML5, CSS Flexbox, Javascript, and SVG are used. 


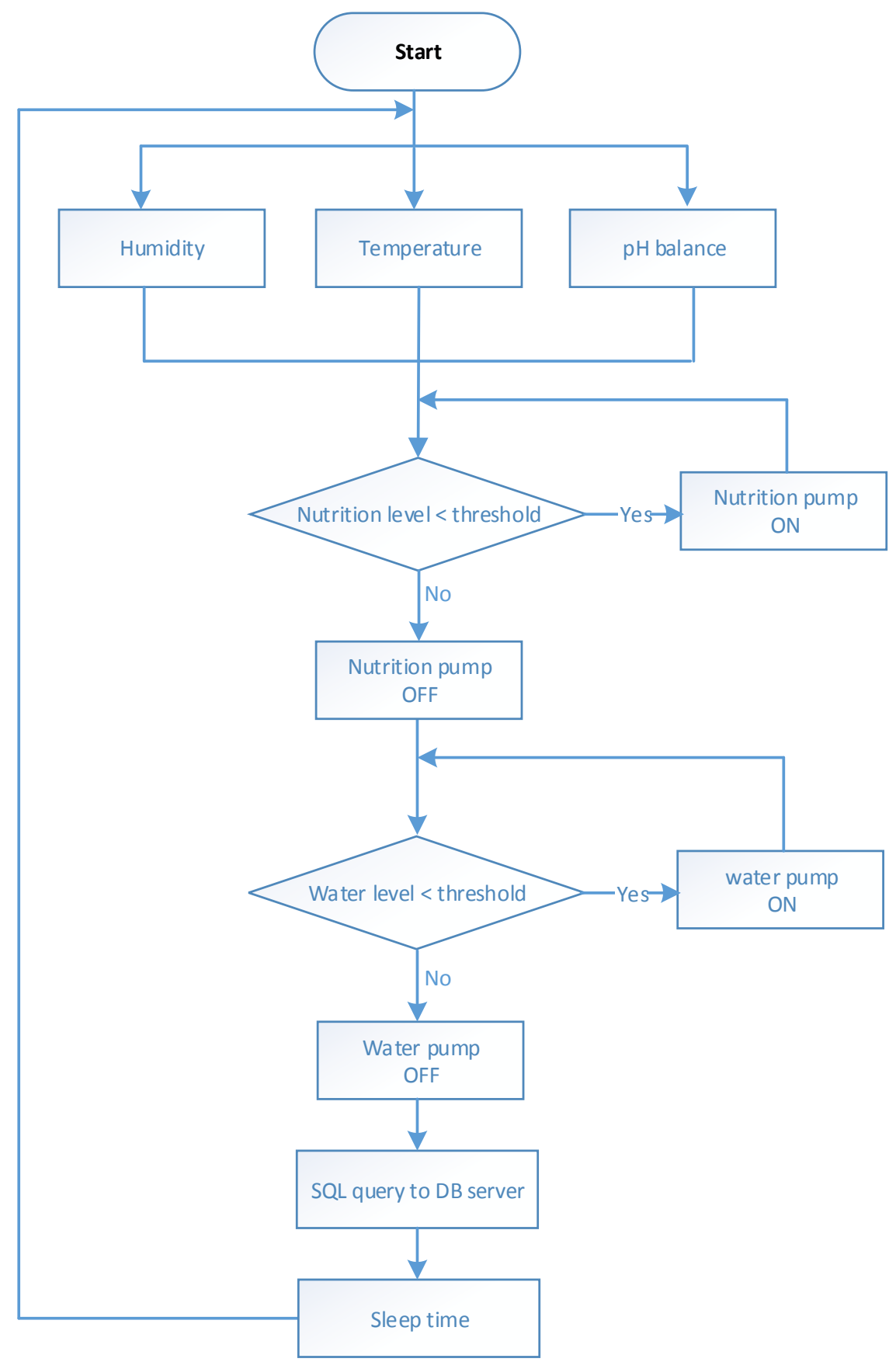

Figure 2. Flow diagram of IoT device

\section{Service Platform}

We designed and implemented the service platform to provide an interface to view the sensor data of the aeroponics system. In figure 3, the service platform provides a web interface to access data using a mobile device 
or client, and provides a database service to save the gathered information from each sensor using IoT devices. For the web service, we installed Apache 2.4.26, MariaDB 10.1.25, and PHP 7.1.7 on Linux system.

\section{IoT device connected on sensors}

We implemented a module to gather each sensors data using IoT devices. Raspberry PI Zero is one of the IoT devices. In this research, we use Raspberry PI Zero to gather information ( $\mathrm{pH}$ balance, temperature, and humidity) from the sensors to control a water pump and dosing pump to add nutrients and water. The gathered information is saved into a database server by sending a SQL query. GPIO pins on the Raspberry PI Zero connects the DHT11 temperature and humidity sensor, Atlas scientific $\mathrm{pH}$ probe and EZO Circuit, water level sensor, LED lights, submersible pump and dosing pumps for the delivery of nutrients. Figure 2 shows the flow chart of the IoT device system.

Each IoT device gathers sensor data (temperature, humidity, and $\mathrm{pH}$ balance) during a certain time. After checking nutrition levels if the nutrition level is below a certain threshold value the Raspberry PI Zero will trigger a relay. The connected dosing pumps start to work to add nutrition in the aeroponics system. If the nutrition level is over a certain threshold value it will stop adding the nutrients in the aeroponics system. The submersible pump process works the same as the dosing pump process.

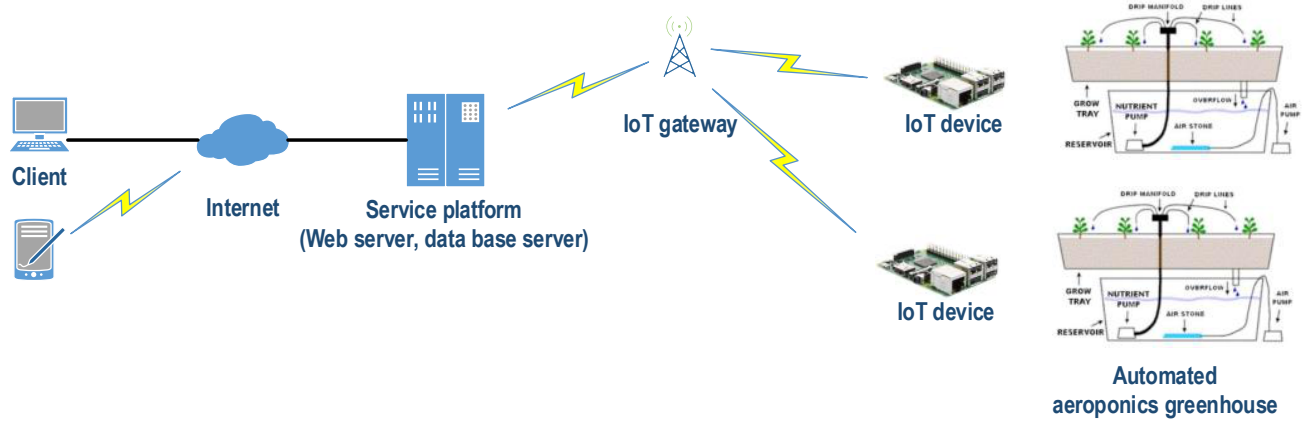

Figure 3. Automated aeroponics system using IoT devices

\section{Conclusion}

In this paper, we have presented an automated aeroponics system using IoT devices. We designed and implemented an automated aeroponics system to collect each sensor's data in the system. We used a Raspberry Pi Zero and designed the system to measure and control the environmental factors necessary to reach our optimal agricultural needs. We implemented a mobile application, service platform, and a control module on an IoT device that the 
user can monitor and control the Aeroponics system remotely. Our proposed system is expected to be a promising application to help farmers increase the production of organic crops in a smart farming system.

\section{References:}

1. K. Ashton, "That 'Internet of Things' Thing," RFiD Journal, vol. 22, no. 7, pp. 97-114, July 2009.

2. M. Ryu, J. Yun, T. Miao, I. Y. Ahn, S. C. Choi and J. Kim, "Design and implementation of a connected farm for smart farming system," 2015 IEEE SENSORS, Busan, 2015, pp. 1-4.

3. T. Qiu, H. Xiao and P. Zhou, "Framework and case studies of intelligence monitoring platform in facility agriculture ecosystem," 2013 Second International Conference on Agro-Geoinformatics (Agro-Geoinformatics), Fairfax, VA, 2013, pp. 522-525.

4. A.Kamilaris, F. Gao, F. X. Prenafeta-Boldu and M. I. Ali, "Agri-IoT: A semantic framework for Internet of Things-enabled smart farming applications," 2016 IEEE 3rd World Forum on Internet of Things (WFIoT), Reston, VA, 2016, pp. 442-447.

5. R. K. Kodali, V. Jain and S. Karagwal, "IoT based smart greenhouse," 2016 IEEE Region 10 Humanitarian Technology Conference (R10HTC), Agra, 2016, pp. 1-6.

6. C. Cambra, S. Sendra, J. Lloret and L. Garcia, "An IoT serviceoriented system for agriculture monitoring," 2017 IEEE International Conference on Communications (ICC), Paris, France, 2017, pp. 1-6.

7. C. Perera, C. H. Liu, S. Jayawardena and M. Chen, "A Survey on Internet of Things From Industrial Market Perspective," in IEEE Access, vol. 2, no., pp. 1660-1679, 2014.

8. P. Lottes, R. Khanna, J. Pfeifer, R. Siegwart and C. Stachniss, "UAVbased crop and weed classification for smart farming," 2017 IEEE International Conference on Robotics and Automation (ICRA), Singapore, 2017, pp. 3024-3031.

9. P. Nintanavongsa, W. Yaemvachi and I. Pitimon, "A self-sustaining unmanned aerial vehicle routing protocol for smart farming," 2016 International Symposium on Intelligent Signal Processing and Communication Systems (ISPACS), Phuket, 2016, pp. 1-5.

10. F. Viani, M. Bertolli and A. Polo, "Low-Cost Wireless System for Agrochemical Dosage Reduction in Precision Farming," in IEEE Sensors Journal, vol. 17, no. 1, pp. 5-6, Jan.1, 12017. 